SNUST 030601

hep-th/0306148

\title{
Can Branes Travel Beyond CTC Horizon in Gödel Universe?*
}

\author{
Yasuaki Hikida ${ }^{a}$ and Soo-Jong Rey ${ }^{a, b}$ \\ School of Physics \& BK-21 Physics Division \\ Seoul National University, Seoul 151-747 KOREA ${ }^{a}$ \\ School of Natural Sciences, Institute for Advanced Study \\ Einstein Drive, Princeton NJ 08540 USA $^{b}$ \\ hikida@phya.snu.ac.kr sjrey@gravity.snu.ac.kr
}

\begin{abstract}
Gödel universe in M-theory is a supersymmetric and homogeneous background with rotation and four-form magnetic flux. It is known that, as seen in inertial frame of co-moving observer, all geodesics with zero orbital angular momentum orbit inside 'surface of light velocity' (CTC horizon). To learn if other probes can travel beyond the CTC horizon, we study dynamics of M-graviton and, in particular, M2-brane, whose motion is affected by Lorentz force exerted by the four-form magnetic flux and by nonzero orbital angular momentum. Classically, we find that both probes gyrate closed orbits, but diameter and center of gyration depends on sign and magnitude of probe's energy, charge and orbital angular momentum. For M2-brane, orbits in general travel outside the CTC horizon. Quantum-mechanically, we find that wave function and excitation energy levels are all self-similar. We draw analogy of probe's dynamics with Landau problem for charged particle in magnetic field.
\end{abstract}

\footnotetext{
${ }^{*}$ Work supported in part by the KOSEF Interdisciplinary Research Grant 98-07-02-07-01-5 and the KOSEF Leading Scientist Grant.
} 
- Albert Einstein

For us believing physicists, the distinction between the past, the present, and the future is only an illusion.

\section{Introduction}

The Gödel universe [1] is a homogeneous space-time with pressureless matter and negative cosmological constant, featured by rotation relative to the local inertial frame associated with each co-moving observer, and, as a result of it, no "absolute" time function ${ }^{\dagger}$, the latter meaning that the space-time does not admit a foliation by globally space-like hyper-surfaces. Because of these difficulties, Gödel universe has brought up many puzzling issues in Einstein's general relativity: the space-time displays closed null curves (CNCs) and closed time-like curves (CTCs), Cauchy problem is ill-defined, and, for quantum field theories defined on it, no obvious notion of unitary Hamiltonian evolution exists. These issues are all concerned with the notion of chronology, on which Hawking has put forward so-called "chronology protection conjecture" (CPC) 2].

String theory and M-theory admit the Gödel universe as a super-symmetric solution [3,4], where, quite importantly, requisite 4-form field strengths are also turned on. It is in this setting that one might hope to gain a better understanding of the above conceptual puzzles and shed light on an eventual resolution. With such motivations, recently, several works have revisited the issue of chronology and (stringy version of) CPC [5, 6, 7, 8,9 . Among them, Boyda et.al. [9] claimed that the chronology is well definable once the Gödel universe is prescribed with the macroscopic holography [10,11]. In effect, their proposal argues for keeping (a part of) the causal region associated with a co-moving observer and replacing the rest (which includes the CTC region) by an observer-dependent holographic screen. Most recently, Drukker et.al. [12] have studied dynamics of BPS super-tube (cylindrical D2-brane supported by fundamental string and D0-brane charges) in Type IIA Gödel universe, and claimed that the super-tube develops an instability in the CTC region despite being a BPS configuration.

To draw some insight concerning the CTCs in the Gödel universe, in this work, we introduce a probe M-graviton and M2-brane and study their dynamics. Geodesics in the Gödel universe was studied previously [13,9. There, it was noted that null and time-like geodesic motions trace gyration orbits whose diameter is set by the energy, and most notably, none of these geodesics never get into the CTC region. An interpretation based on the result, which seems to be implicit in the holographic proposal of [9], may be that the CTC region, though it exists, is never reachable by a stationary co-moving observer.

An immediate question is whether the feature that geodesic orbits never get into the CTC region holds also for all other available probes in M-theory. To answer this question, in this

\footnotetext{
${ }^{\dagger}$ Technically, a "time function" is a smooth, differentiable scalar function $t(x)$ such that $\left(\partial_{m} t\right)\left(\partial^{m} t\right)>0$ everywhere in space-time.
} 
work, we study classical and quantum dynamics of a probe M-graviton and, in particular, M2brane in the Gödel universe. The reason why M2-brane might serve as a useful probe for the question posed is because of the following intuitive argument. In addition to the rotating spacetime metric, the Gödel universe in M-theory is characterized by constant 4-form magnetic field strengths, whose strength is set by the strength of the rotation. M2-brane is an electrically charged object coupled minimally to the 4-form field, so it would experience the 4-form Lorentz force in addition to the geodesic force acted upon by the Gödel metric. This means that, under suitable circumstances (which we will spell out explicitly later), the Lorentz force might well cancel the geodesic force. In that case, M2-brane's orbit can be larger than the null geodesics, and would eventually be able to travel into the region where co-moving observers perceive CTCs present.

We find it useful to compare the situation with the well-known Landau problem: dynamics of a charged particle on a homogeneous 2-space (such as sphere $\mathbf{S}^{2}$, plane $\mathbf{R}^{2}$, and pseudo-sphere $\mathbf{H}^{2}$ ) under a uniform magnetic field. Take, for definiteness, the BPS particle $m=|q|$ on a plane $\mathbf{R}^{2}$. On $\mathbf{R}^{2}$, geodesics are straight lines. The magnetic field exerts Lorentz force to the BPS particle, causing it to undergo gyration around the Larmor orbit, whose radius of curvature is set by initial velocity $\mathbf{v}$ as

$$
R_{\mathrm{L}}=\left(\frac{c}{B}\right)|\mathbf{v}|
$$

Quantum mechanically, the particle dynamics is describable as that of two-dimensional simple harmonic oscillator, whose energy spectrum is given in terms of non-negative quantum number $\mathbf{n}$ as

$$
E_{\mathrm{L}}=\Omega_{\mathrm{L}}\left(\mathbf{n}+\frac{1}{2}\right) \quad \text { where } \quad \Omega_{L}=\left(\frac{B}{c}\right) .
$$

As $\mathbf{R}^{2}$ is a homogeneous space, the gyration takes place isomorphically everywhere and its center can be brought to, say, the center $O$ by Killing transformations. Dynamics on $\mathbf{S}^{2}$ or $\mathbf{H}^{2}$ is further corrected by curvature-dependent contribution $\Delta E_{\mathrm{L}}= \pm \frac{1}{2 R^{2}}\left(\mathbf{n}+\frac{1}{2}\right)^{2}$ to the energy spectrum Eq.(1.2), where $R$ is the radius of curvature, but otherwise qualitatively the same. In particular, gyration orbit is closed for strong magnetic field and its center can be brought to to the center $\mathcal{O}$ by Killing transformations.

This paper is organized as follows. In section 2, we investigate classical dynamics of a probe brane in one of the simpler Gödel universes in M-theory, $\mathcal{G}_{5} \times X_{6}$. We study both M-graviton and M2-brane probes, and show that M2-brane orbit can extend beyond the CTC horizon. In section 3, we study quantum dynamics of these probe branes. We find that the energy spectrum is discrete and is strikingly reminiscent of that of the Landau problem, Eq.(1.2). Section 4 is devoted to discussion of various points worthy of further investigation. 


\section{Classical Dynamics}

\subsection{Set-Up}

The Gödel universe in M-theory is a family of classical vacua of the form $\mathcal{G}_{2 n+1} \times X_{10-2 n}$, 4], where the index $n=1, \cdots, 5$ refers to the number of rotating planes. The simplest situation in which multiple planes are rotating simultaneously is $\mathcal{G}_{5} \times X_{6}$, and we shall focus our consideration mainly to this case in this work ${ }^{\ddagger}$. The metric of $\mathcal{G}_{5} \times X_{6}$ is given by

$$
\begin{aligned}
\mathrm{d} s_{\mathrm{M}}^{2} & =-(\mathrm{d} t+\mu \omega)^{2}+\mathrm{d} s^{2}\left(\mathbf{R}^{4}\right)+\mathrm{d} s^{2}\left(X_{6}\right) \\
G_{4} & \equiv \mathrm{d} C_{3}=2 \mu J \wedge K,
\end{aligned}
$$

where $\omega$ is the twist one-form on the spatial slice $\mathbf{R}^{4}$ in $\mathcal{G}_{5}$, while $J, K$ are Kähler two-forms on $\mathbf{R}^{4}$ and a six-dimensional 'internal' manifold $X_{6}$, respectively. Locally, the one-form $\omega$ is related to $J$ as $J=\mathrm{d} \omega$. The constant $\mu$ is a parameter for the simultaneous rotation of $\mathbf{R}^{2} \oplus \mathbf{R}^{2} \subset \mathbf{R}^{4}$ as well as for the 4-form magnetic flux $G_{4} \equiv \mathrm{d} C_{3}$. It can be set to unity by rescaling proper distance on $\mathbf{R}^{4}$ by $1 /|\mu|$, but, for the consideration of dimensional analysis and (pseudo)-symmetry transformation, we find it useful to retain it explicitly.

We introduce a probe M2- or $\overline{\mathrm{M} 2}$-brane, which is coupled minimally to the 3 -form potential $C_{3}$, and examine classical dynamics of it in the background Eq.(2.1). Classical dynamics of the probe brane is governed by the Born-Infeld action

$$
S=-T_{2} \int_{\Sigma_{3}} \sqrt{-\operatorname{det} X^{*} g}-Q \int_{\Sigma_{3}} X^{*} C_{3}
$$

where $X^{*} g, X^{*} C_{3}$ are the pull-backs of the metric and the 3-form potential in Eq.(2.1) on the world-volume of M2-brane. Noether charge of the M2-brane is denoted as $Q$, measured in unit of the M2-brane tension $T_{2}$. Thus, for a BPS M2- or $\overline{\mathrm{M} 2}$-brane, $Q=T_{2}$ or $Q=-T_{2}$, respectively.

For definiteness, consider a M2-brane wrapped on a supersymmetric two-cycle $\Sigma$ in $X_{6}$ §. Rigid dynamics of the M2-brane is describable by dimensionally reducing Eq.(2.1) to fivedimensional Gödel universe $\mathcal{G}_{5}$ threaded with the magnetic flux $G_{2} \equiv \oint_{\Sigma} G_{4}$ (up to normalization):

$$
\begin{aligned}
\mathrm{d} s_{5}^{2} & =-(\mathrm{d} t+\mu \omega)^{2}+\mathrm{d} s^{2}\left(\mathbf{R}^{4}\right) \\
G_{2} & \equiv \mathrm{d} C_{1}=2 \sqrt{3} \mu J
\end{aligned}
$$

\footnotetext{
†The simplest Gödel universe, $\mathcal{G}_{3} \times X_{8}$, involving a single rotating plane preserves 8 supersymmetries only, as contrasted to those involving multiple rotating planes, which preserve $16+4=20$ supersymmetries $(n=2,3,4)$ or $16+2=18$ supersymmetries $(n=5)$.

$\S$ The analysis of [4] indicates that this is a supersymmetric configuration.
} 
and the M2-brane reduces to a charged point-particle of mass $m=T_{3} \operatorname{vol}(\Sigma)$ and electric charge $q=Q \operatorname{vol}(\Sigma)$, coupled minimally to the 'vector' potential $C_{1}$. From Eq.(2.2), the effective worldline action of the 5-dimensional charged particle is given in local reparametrization invariant way as

$$
S=\int \mathrm{d} \tau\left[\frac{1}{2}\left(e^{-1} g_{m n}(x) \dot{x}^{m} \dot{x}^{n}-e m^{2}\right)-\frac{q}{2 \sqrt{3}} C_{1}(x) \dot{x}^{m}\right], \quad(m, n=0,1, \cdots, 4)
$$

where $\dot{x}^{m} \equiv \mathrm{d} x^{m} / \mathrm{d} \tau$. We solve the equation of motion for $x^{m}(\tau)$ :

$$
\frac{D \dot{x}^{m}}{D \tau}-\frac{\dot{e}}{e} \dot{x}^{m}+e \frac{q}{2 \sqrt{3}} G_{2}^{m} \dot{x}^{n}=0
$$

where $D / D \tau$ refers to the covariant derivative with respect to the Christoffel connection $\Gamma_{n p}^{m}$ of the metric Eq.(2.3) . Equation of motion for the worldline einbein $e(\tau)$ is

$$
e(\tau)=m^{-1} \sqrt{-g_{m n}(x) \dot{x}^{m} \dot{x}^{n}}
$$

The worldline action Eq.(2.4) is invariant under local reparametrization invariance, $\tau \rightarrow f(\tau)$ and $e \rightarrow e /|\dot{f}(\tau)|$, and we fix it by choosing the gauge $e=+1$, so that $\tau$ is the affine parameter of the world-line. The gauge-fixed equations of motion for $x^{m}(\tau)$ are

$$
\frac{D \dot{x}^{m}}{D \tau}+\frac{q}{2 \sqrt{3}} G_{2}^{m} \dot{x}^{n}=0
$$

subject to the constraint Eq.(2.5).

To proceed further, we find it convenient to express the metric Eq.(2.3) in the bipolar coordinates (see, e.g., Eq.(3.7) in [9]) as:

$$
\begin{aligned}
\mathrm{d} s_{5}^{2}= & -\mathrm{d} t^{2}-2 \mu\left(r_{1}^{2} \mathrm{~d} \phi_{1}+r_{2}^{2} \mathrm{~d} \phi_{2}\right) \mathrm{d} t-2 \mu^{2} r_{1}^{2} r_{2}^{2} \mathrm{~d} \phi_{1} \mathrm{~d} \phi_{2} \\
& +r_{1}^{2}\left(1-\mu^{2} r_{1}^{2}\right) \mathrm{d} \phi_{1}^{2}+r_{2}^{2}\left(1-\mu^{2} r_{2}^{2}\right) \mathrm{d} \phi_{2}^{2}+\mathrm{d} r_{1}^{2}+\mathrm{d} r_{2}^{2}
\end{aligned}
$$

and the one-form connection $C_{1}$ in the Landau gauge as:

$$
G_{2} \equiv \mathrm{d} C_{1}=2 \sqrt{3} \mu \mathrm{d}\left(r_{1}^{2} \wedge \mathrm{d} \phi_{1}+r_{2}^{2} \wedge \mathrm{d} \phi_{2}\right)
$$

We refer the origin of the coordinate system as $O$. Notice that the bipolar coordinates Eq.(2.7) displays explicitly that the signature of $\phi_{1,2}$ line element flips sign across the hyper-surface $\sqrt{r_{1}^{2}+r_{2}^{2}}=1 /|\mu|$. Thus, at the hyper-surface, paths around $\phi_{1}$ or $\phi_{2}$ are CNCs, and, beyond the hyper-surface, they are CTCs. As such, we will refer these codimension-one hyper-surfaces located at

$$
R_{\mathrm{CTC}}=\frac{1}{|\mu|}
$$


as "CTC horizon". We emphasize again that the "CTC horizon" is a notion set forth specifically by each co-moving observer, whom we have put conveniently at the origin $O$. As Gödel universe is a homogeneous space, co-moving observers placed at any location on $G_{5}$ are all equivalent and can be always brought to the origin $O$.

Using explicit form of the Christoffel connections as recollected in the appendix, we find the equations of motion Eq.(2.6) are given by

$$
\begin{aligned}
& \ddot{t}+2 \mu^{2} r_{1} \dot{r}_{1} \dot{t}+2 \mu^{3} r_{1}^{3} \dot{\phi}_{1} \dot{r}_{1}+2 \mu^{3} r_{1} r_{2}^{2} \dot{\phi}_{2} \dot{r}_{1}+2 q \mu^{2} r_{1} \dot{r}_{1}+(1 \leftrightarrow 2)=0, \\
& \ddot{r}_{1}+2 \mu r_{1} \dot{\phi}_{1} \dot{t}-r_{1}\left(1-2 \mu^{2} r_{1}^{2}\right) \dot{\phi}_{1}^{2}+2 \mu^{2} r_{1} r_{2}^{2} \dot{\phi}_{1} \dot{\phi}_{2}+2 q \mu r_{1} \dot{\phi}_{1}=0, \\
& \ddot{\phi}_{1}-\frac{2 \mu}{r_{1}} \dot{r}_{1} \dot{t}+2\left(\frac{1}{r_{1}}-\mu^{2} r_{1}\right) \dot{\phi}_{1} \dot{r}_{1}-\frac{2 \mu^{2} r_{2}^{2}}{r_{1}} \dot{\phi}_{2} \dot{r}_{1}-2 q \mu \frac{\dot{r}_{1}}{r_{1}}=0,
\end{aligned}
$$

and similar ones with $(1 \leftrightarrow 2)$ for the latter two equations of motion.

The Gödel universe Eq.(2.3) or, equivalently, Eqs.(2.7] 2.8] exhibits the following symmetries:

$$
\begin{aligned}
\mathrm{P} & : \quad \omega \rightarrow-\omega \quad \text { and } \quad \mu \rightarrow-\mu \\
\mathrm{PT} & : \quad t \rightarrow-t, \quad \mu \omega \rightarrow-\mu \omega \text { and } q \rightarrow-q .
\end{aligned}
$$

Notice that $\omega \rightarrow-\omega$ in Eq.(2.1) amounts in bipolar coordinates to parity inversion, $\phi_{1,2} \rightarrow$ $-\phi_{1,2}$, in each $\mathbf{R}^{2}$-plane. Thus, Eq.(2.13) acts as parity inversion accompanied by inversion of the rigid rotation, while Eq.(2.14) does as simultaneous action of the time-reversal and parity-inversion on $\mathcal{G}_{5}$ and $X_{6}$. We will find later these (pseudo)symmetries serve useful for understanding classical and quantum dynamics of the probe objects.

As the metric and the magnetic field strength on $\mathcal{G}_{5}$ are functions of $r_{1}, r_{2}$ only, $-\partial_{t}, \partial_{\phi_{1}}$, $\partial_{\phi_{2}}$ are Killing vectors. Accordingly, the canonical momenta conjugate to $-t, \phi_{1}$ and $\phi_{2}$ are conserved, first integrals of motion. They are

$$
\begin{aligned}
E & =\dot{t}+\mu\left(r_{1}^{2} \dot{\phi}_{1}+r_{2}^{2} \dot{\phi}_{2}\right) \\
L_{1} & =r_{1}^{2} \dot{\phi}_{1}-\mu^{2} r_{1}^{2}\left(r_{1}^{2} \dot{\phi}_{1}+r_{2}^{2} \dot{\phi}_{2}\right)-\mu r_{1}^{2}(\dot{t}+q) \\
L_{2} & =r_{2}^{2} \dot{\phi}_{2}-\mu^{2} r_{2}^{2}\left(r_{1}^{2} \dot{\phi}_{1}+r_{2}^{2} \dot{\phi}_{2}\right)-\mu r_{2}^{2}(\dot{t}+q) .
\end{aligned}
$$

Inverting these relations,

$$
\begin{aligned}
\dot{\phi}_{1} & =\frac{L_{1}}{r_{1}^{2}}+\mu(E+q) \\
\dot{\phi}_{2} & =\frac{L_{2}}{r_{2}^{2}}+\mu(E+q) \\
\dot{t} & =E-\mu^{2}\left(r_{1}^{2}+r_{2}^{2}\right)(E+q)-\mu\left(L_{1}+L_{2}\right) .
\end{aligned}
$$


In these equations, the first terms are standard. The second term in Eq.(2.18) reflect frame dragging rotation of the Gödel universe. The last term in Eq.(2.18), depending on $L_{1}, L_{2}$, is a sort of 'spin-orbit' coupling between frame-dragging rotation and probe's orbital angular momenta.

Recall that, using homogeneity of $\mathcal{G}_{5}$, any location can be brought to the origin $O\left(r_{1}=\right.$ $r_{2}=0$ in the bipolar coordinates Eq.(2.7) by a sequence of Killing transformations. Thus, we shall be considering a co-moving observer located at the origin $O$, and let the observer perform experiments for exploring causal structure of the Gödel universe by sending off the probe objects available such as M-graviton or M2-branes. We will study first the probes with zero angular momenta, and then those with nonzero angular momenta. In both cases, wherever relevant, we draw close analogy with the well-known results of the Landau problem Eqs. (1.1. 1.2).

\subsection{Probes with Zero Angular Momenta}

We will first consider $L_{1}=L_{2}=0$ case. In this case, there is no centrifugal barrier, and the probe brane passes through the origin, where the co-moving observer is located. We then obtain the radial equations of motion as exerting harmonic oscillations isomorphically on each $\mathbf{R}^{2}$-plane:

$$
\ddot{r}_{1}+\mu^{2}(E+q)^{2} r_{1}=0 \quad \text { and } \quad \ddot{r}_{2}+\mu^{2}(E+q)^{2} r_{2}=0
$$

subject to the gauge-fixing constraint, coupling the motion on the two $\mathbf{R}^{2}$-planes,

$$
\left\{\dot{r}_{1}^{2}+(E+q)^{2} \mu^{2} r_{1}^{2}\right\}+\left\{\dot{r}_{2}^{2}+(E+q)^{2} \mu^{2} r_{2}^{2}\right\}=\left(E^{2}-m^{2}\right)
$$

With the initial condition $r_{1}(0)=r_{2}(0)=0$ that the probe starts from co-moving observer's location $O$, the solution is

$$
\begin{aligned}
\phi_{1}(\tau) & =\mu(E+q) \tau+\phi_{1}^{(0)} \\
\phi_{2}(\tau) & =\mu(E+q) \tau+\phi_{2}^{(0)} \\
r_{1}(\tau) & =\frac{\sqrt{E^{2}-m^{2}}}{|\mu(E+q)|} \sin (|\mu(E+q)| \tau) \cos \theta \\
r_{2}(\tau) & =\frac{\sqrt{E^{2}-m^{2}}}{|\mu(E+q)|} \sin (|\mu(E+q)| \tau) \sin \theta \\
t(\tau) & =\left(E-\frac{1}{2}\left(\frac{E^{2}-m^{2}}{E+q}\right)\right) \tau+\frac{1}{4 \mu} \frac{E^{2}-m^{2}}{(E+q)^{2}} \sin (2|\mu(E+q)| \tau)+t^{(0)} .
\end{aligned}
$$

Here, $\theta(0 \leq \theta \leq \pi / 2)$ parametrizes projection of the motion to each $\mathbf{R}^{2}$-plane, $\phi_{1}^{(0)}, \phi_{2}^{(0)}, t^{(0)}$ are parameters fixed by initial condition, and the conserved energy $E$, Eq.(2.15), ranges over $E \geq m$ 


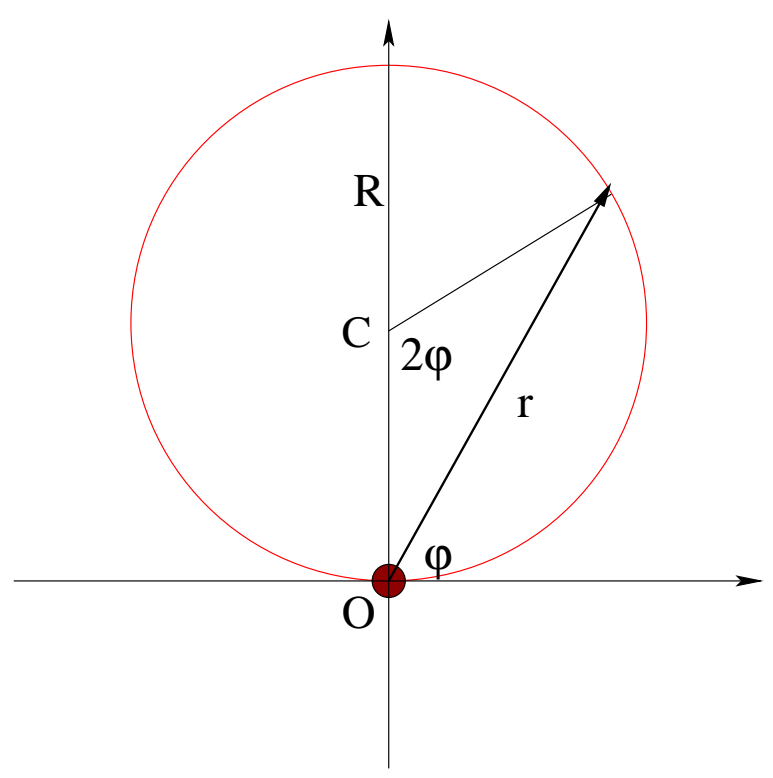

Figure 1: Trajectory of the probe with zero angular momenta, passing through co-moving observer located at $O$. The probe traces the Larmor orbit, whose radius is $R$ and gyration speed is $\sqrt{E^{2}-m^{2}}$.

or $E \leq-m$ (to yield physically meaningful solution satisfying Eq.(2.19)). In our convention, $E, m$ and $q$ carries mass dimension 1 , and BPS conditions set inequalities $|E| \geq m \geq|q|$. We thus see that probe's trajectory is uni-directional: angular velocities $\dot{\phi}_{1}(\tau), \dot{\phi}_{2}(\tau)$ revolve the same direction as the background Gödel universe Eq.(2.7) for $\operatorname{sgn}(\mu(E+q))>0$ and opposite direction for $\operatorname{sgn}(\mu(E+q))<0$, respectively . Under PT-conjugation of Eq.(2.14), the anti-probe follows the same trajectory but in opposite directions.

The motion is reminiscent of the Landau problem mentioned earlier. As evident from Eq.(2.20), probe's trajectory traces a circular orbit on each $\mathbf{R}^{2}$-plane, as depicted in Fig [1. One may describe the orbit with respect to the center of the orbit $C$ (instead of co-moving observer's location $O$ ). The gyration diameter $D=2 R$, as depicted in Fig 1 is set by the conserved energy

$$
D=2 R=R_{\mathrm{CTC}} \frac{\sqrt{E^{2}-m^{2}}}{|E+q|}
$$

but is constant otherwise. Using trigonometry, gyration velocity $\mathbf{v}$ around $C$ (as measured in affine time) is also determined as

$$
\mathbf{v}=R \cdot 2 \dot{\phi}_{1}=R \cdot 2 \dot{\phi}_{2}=\operatorname{sgn}(\mu(E+q)) \sqrt{E^{2}-m^{2}} .
$$

\footnotetext{
T This feature is also reflected in the quantum dynamics of the probes. See section 3.
} 


\subsubsection{M-Graviton}

For a neutral probe, $q=0$, corresponding to Kaluza-Klein modes of the M-theory graviton, Eq.(2.20) reduces to the geodesics studied in [13,9].

- For the massless mode, $m=0$, the geodesics start out radially from the origin $O$, and sweep out a circular gyration orbit, touching the CTC horizon $R_{\mathrm{CTC}}$ associated with the co-moving observer at $O$. That is,

$$
r_{1}(\tau), r_{2}(\tau) \leq R_{\mathrm{CTC}}
$$

as depicted as the leftmost circles in Fig.2. To reach the CTC horizon, $\phi_{1}, \phi_{2}$ should sweep out $\pi / 2$, so it takes affine time $\Delta \tau=(\pi / 2|\mu E|)$. Consequently, a complete revolution around the orbit takes $\Delta \tau=(\pi /|\mu E|)$ in affine time, and hence from Eq.(2.20),$\Delta t=(\pi / 2|\mu|)$ in co-moving observer's time. Notice that the gyration orbit is independent of graviton's energy $E$. One can see this already from Eq.(2.19) - both the harmonic frequency in the left-hand side and the constant of motion in the right-hand side are proportional to $E^{2}$, and can be rescaled away.

- For the massive modes, the geodesics extend radially only up to the distance

$$
r_{1}, r_{2} \leq R_{\mathrm{CTC}} \sqrt{1-\frac{m^{2}}{E^{2}}}<R_{\mathrm{CTC}}
$$

viz. the projected orbit has a radius smaller than the CTC horizon $R_{\mathrm{CTC}}$ (Recall from Eq.(2.9) that the causal region as perceived by the co-moving observer is separated from the CTC region by the CTC horizon $r_{1,2}=R_{\mathrm{CTC}}$ ). It follows that geodesics of M-theory graviton in the Gödel universe lie entirely within the causal region [13,9] ". Otherwise, the geodesics behave essentially the same as the massless ones. In particular, it takes exactly the same amount of affine and co-moving time for the gyration to undergo a complete revolution.

\subsubsection{M2-branes}

For $q \neq 0$, corresponding to black M2-brane, the trajectory Eq.(2.20) entails several new features. Because of the background magnetic field, the M2-branes, being an electrically charged probe, experiences the Lorentz force in addition to the force exerted by the Gödel universe rotation. Recalling that the rotational force is uni-directional, depending on the sign of M2brane charge, the Lorentz force may act to add up to or to cancel off the rotational force. It clearly points to the possibility that the co-moving observer can arrange a probe brane that travels beyond the CTC horizon and comes back. Let us analyze how this may happen.

\footnotetext{
${ }^{\|}$Taking up this observation, in [9], it was proposed to cut off the region outside the CTC horizon, paste it with flat space-time, and place the holographic screen inside the causal region, where the area of the holographic screen as prescribed in [10 11] is maximized.
} 




Figure 2: Trajectories of various probes. The inner circle refers to the CTC horizon as seen in the inertial frame of comoving observer at the center, outside which closed time-like curves are present. Geodesics of $M$-graviton, shown in the left for three different angular initial conditions, are confined inside the CTC horizon. Trajectory of M2-branes with $E>0$ are shown in the right for $q>0$ and $q<0$, respectively. Notice that $q>0$ orbit is always inside the CTC horizon, while $q<0$ orbit extends beyond the CTC horizon.

Eq.(2.20) indicates that the net effect depends crucially on the relative sign between the energy $E$ and the charge $q$. Consider an M2-brane with $E>m$, orbiting through co-moving observer's location $O$. Diameter $D$ of the orbit is given by Eq.(2.21), so let us examine how $D$ depends on $E$ and $q$. We are particularly interested in whether $D$ could be larger than $R_{\mathrm{CTC}}$ for appropriate values of $E$ and $q$. We see from Eq.(2.20) that, for BPS M2-brane with positive charge $q=m$,

$$
D_{\text {sub }}=R_{\mathrm{CTC}} \sqrt{(E-m) /(E+m)}<R_{\mathrm{CTC}} \quad \text { for } \quad \operatorname{sgn}(E q)>0,
$$

so the orbit gyrates entirely inside the CTC horizon of the co-moving observer $O$. We will refer the trajectory as "sub-gyration orbit" of diameter $D_{\text {sub. }}$ For BPS M2-brane with negative charge $q=-m$,

$$
D_{\text {super }}=R_{\mathrm{CTC}} \sqrt{(E+m) /(E-m)}>R_{\mathrm{CTC}} \quad \text { for } \quad \operatorname{sgn}(E q)<0,
$$

so, as observed by the co-moving observer $O$, the orbit passes through the CTC horizon and gyrate into the region where CTCs are present! This is in stark contrast to the situation of M-graviton geodesics studied in the previous section. We will refer the trajectory as "supergyration orbit" of diameter $D_{\text {super }}$. The two types of behavior are depicted as the rightmost two orbits in Fig, 2 ,

Information concerning M2-brane's dynamics for $E<-m$ are obtainable by applying the PT conjugation Eq.(2.14). One finds that the two types of behavior are interchanged: with $E<-m$, BPS M2-brane with positive charge $q=m$ traces "super-gyration orbit" and extends beyond the CTC horizon, while M2-brane with negative charge $q=-m$ traces "sub-gyration orbit" and remains inside the CTC horizon. 
Let us draw further comparison between "sub-gyration orbits" and "super-gyration orbits". We consider the positive-energy branch $E>m$ only, as the negative-energy branch $E<-m$ is obtainable by PT conjugation of the positive-energy branch.

- Diameter of the sub-gyration orbit is a monotonically increasing function of $E$, ranging over $0<D_{\text {sub }}<R_{\mathrm{CTC}}$. In contrast, diameter of the super-gyration orbit is a monotonically decreasing function of $E$, ranging over $R_{\mathrm{CTC}}<D_{\text {super }}<\infty$. Interestingly, in both cases, orbits at extreme high-energy limit accumulate to the CTC horizon, viz. the 'velocity of light surface', and the gyration velocity becomes infinite. In other words, in so far as dynamics of M-theory probes is concerned, the CTC horizon defines a universal infinite-momentum light-front frame, as gyration orbits of both the M-graviton and M2-brane asymptote all to it as the "velocity of light' surface. This suggests a viable microscopic holography of the Gödel universe in Mtheory [14.

- From $t$-velocity Eq.(2.18), we see that, for $L_{1,2}=0$ under consideration, the co-moving observer at $O$ would draw the standard interpretation of forward time-flow for $E>m$ and backward time-flow for $E<-m$. Far away from $O$, however, Eq.(2.18) indicates that the time-flow would be seen reversed. Using the result Eq.(2.20), one finds that the reversal takes place at a distance $r_{\mathrm{r}}$ :

$$
r_{\mathrm{r}}=R_{\mathrm{CTC}} \sqrt{\frac{E}{E+q}} .
$$

Comparing this with the diameter $D$ of the gyration orbit Eq.(2.21), we find that M2-branes whose energy and charge have same sign would never reverse the time-flow, but M2-branes whose energy and charge have opposite sign would do so. At the moment of time-flow reversal, angular position $\phi_{1,2}$ of the probe M2-brane is at $\sin \phi_{\mathrm{r}}=\sqrt{E /(E+|q|)}$.

\subsection{Probes with Nonzero Orbital Angular Momenta}

So far, we assumed that the probe carries no angular momentum. In case the angular momenta $L_{1}, L_{2}$ are nonzero, new intriguing features arise.

- Probe's orbits all migrate off the location of the co-moving observer, and, if the orbital angular momentum meets a suitable condition, penetrate into the CTC region. This is readily seen as follows. Substitute Eq.(2.16), (2.17) and (2.18) into (2.5) with $e=1$. We then obtain

$$
\left(\dot{r}_{1}\right)^{2}+\left(\dot{r}_{2}\right)^{2}=\left(E^{2}-m^{2}\right)-V_{\text {eff }}\left(r_{1}, r_{2}\right)
$$

where

$$
V_{\mathrm{eff}}\left(r_{1}, r_{2}\right)=\left(\frac{L_{1}}{r_{1}}+\mu r_{1}(E+q)\right)^{2}+\left(\frac{L_{2}}{r_{2}}+\mu r_{2}(E+q)\right)^{2} .
$$


The gyration orbits now range over $V_{\text {eff }}\left(r_{1}, r_{2}\right) \leq\left(E^{2}-m^{2}\right)$, so

$$
\begin{aligned}
& \left(\frac{L_{1}}{r_{1}}+\mu r_{1}(E+q)\right)^{2} \leq\left(E^{2}-m^{2}\right) \cos ^{2} \theta \\
& \left(\frac{L_{2}}{r_{2}}+\mu r_{2}(E+q)\right)^{2} \leq\left(E^{2}-m^{2}\right) \sin ^{2} \theta .
\end{aligned}
$$

Consider the positive-energy branch, $E>m^{* *}$. We see from the above inequalities that not all angular momenta are physically allowed. Those with $L_{1}, L_{2}<0$ are always possible, but those with $L_{1}, L_{2}>0$ cannot be arbitrarily large. Rather, angular momenta are bounded by

$$
-\infty<\left(L_{1}, L_{2}\right)<L_{\max }(\cos \theta, \sin \theta) \quad \text { where } \quad L_{\max } \equiv \frac{1}{4} R_{\mathrm{CTC}}(E-q) .
$$

In this case, the classical turning points are

$$
\begin{aligned}
& r_{1}^{( \pm)}=\frac{D}{2}\left|\cos \theta \pm \sqrt{\cos ^{2} \theta-\frac{L_{1}}{L_{\max }}}\right| \\
& r_{2}^{( \pm)}=\frac{D}{2}\left|\sin \theta \pm \sqrt{\sin ^{2} \theta-\frac{L_{2}}{L_{\max }}}\right| .
\end{aligned}
$$

- Intuitively, one expects that orbital angular momentum affects the orbits similar to the Lorentz force. The actual effect is rather interesting. For $L_{1}, L_{2}>0$, the gyration center remains unchanged from that for zero angular momentum, viz. located when projected on each $\mathbf{R}^{2}$-plane at distance $\frac{1}{2} D \cos \theta$ and $\frac{1}{2} D \sin \theta$, respectively. On the other hand, the diameter of each orbit is affected. On each $\mathbf{R}^{2}$-plane, the diameter is $D \sqrt{\cos ^{2} \theta-\left(L_{1} / L_{\max }\right)}$ and $D \sqrt{\sin ^{2} \theta-\left(L_{2} / L_{\max }\right)}$, respectively. So, the gyration orbits shrink once the orbital angular momentum is applied to the probe same direction as the rotation of the Gödel universe background. At and above the critical angular momenta Eq.(2.24), the orbits cease to exist. For $L_{1}, L_{2}<0$, the effects are reversed: the orbit diameter remains unchanged from that for zero orbital angular momenta, viz. projected on each $\mathbf{R}^{2}$-plane, it is $D \cos \theta$ and $D \sin \theta$, respectively, but the gyration center is shifted further away from the co-moving observer $O$. So, once the orbital angular momentum is applied to the probe the opposite direction as the rotation of the Gödel universe background, the orbit slides off from the hand of the co-moving observer. In particular, as perceived by the co-moving observer at $\mathcal{O}$, the orbit passes through the CTC horizon and travels the CTC region.

- The time-flow $\dot{t}$ is now modified further by the 'spin-orbit coupling', the last term in Eq.(2.18), and can be reverted, similar to the situation with the Lorentz force. This is evident for $L_{1}, L_{2}<$ 0 : if magnitude of $L_{1}, L_{2}$ is large enough, gyration center migrates outside the CTC horizon, causing the second term in Eq.(2.18) to dominate over the other terms. For $L_{1}, L_{2}>0$, however,

\footnotetext{
${ }^{* *}$ Again, negative-energy branch, $E<-m$, is deducible by applying PT-conjugation, under which $L_{1,2} \rightarrow$
} $-L_{1,2}$ as well, to the following consideration. 
the coupling cannot revert the time-flow due in part to the limit Eq.(2.24) and the fact that the orbit stays inside the CTC horizon.

\subsection{IIB String Theory Setup}

Instead of uplifting to M-theory, equivalently, to Type IIA string theory, one can uplift the five-dimensional Gödel universe $\mathcal{G}_{5}$ to Type IIB string theory, as originally demonstrated in 6 . The Type IIB background is given by

$$
\begin{aligned}
\mathrm{d} s_{\text {IIB }}^{2} & =g_{m n} \mathrm{~d} x^{m} \mathrm{~d} x^{n}+\left(\mathrm{d} y+\frac{1}{2 \sqrt{3}} A_{m} \mathrm{~d} x^{m}\right)^{2}+\mathrm{d} s^{2}\left(X_{4}\right), \\
H_{3} & =\mathrm{d} C_{2}=\frac{1}{2 \sqrt{3}} \mathrm{~d} A \wedge\left(\mathrm{d} y+\frac{1}{2 \sqrt{3}} A\right)-\frac{1}{2 \sqrt{3}}^{*} \mathrm{~d} A .
\end{aligned}
$$

Here, the ten-dimensional coordinates are represented as $\left(x^{m}, y, z^{a}\right)$ with $m=0, \cdots, 4$ and $a=1, \cdots, 4$. The metric $g_{m n}$ and the gauge connection $A$ are those of maximally supersymmetric, five-dimensional Gödel universe. The Hodge dual * is defined with respect to the five-dimensional metric $g_{m n}$.

For the M-theory uplift, the probe that can couple to the background magnetic flux in the five-dimensional Gödel universe was the M2-brane wrapped on two-cycle $\Sigma_{2}$ in the sixdimensional 'internal' space $X_{6}$. For the Type IIB string theory uplift, a viable probe that can couple to the background 3-form flux Eq.(2.27) in the five-dimensional Gödel universe is the Kaluza-Klein mode along the 6-th dimension (whose coordinates are labelled as $y$ in Eq.(2.26) ).

Consider null geodesics in 6-dimensional Gödel universe Eq.(2.26). Repeating the same analysis as in section 2.1, one finds the conserved, first integrals of motion:

$$
\begin{array}{ccc}
E & =\dot{t}+\mu\left(r_{1}^{2} \dot{\phi}_{1}+r_{2}^{2} \dot{\phi}_{2}\right) & P_{y}=-\dot{y}-\mu\left(r_{1}^{2} \dot{\phi}_{1}+r_{2}^{2} \dot{\phi}_{2}\right) \\
L_{1}=r_{1}^{2} \dot{\phi}_{1}-\mu r_{1}^{2}(\dot{t}-\dot{y}) & L_{2}=r_{2}^{2} \dot{\phi}_{2}-\mu r_{2}^{2}(\dot{t}-\dot{y}) .
\end{array}
$$

One then finds that solution of the null geodesics is precisely the same as Eq.(2.20) provided the conserved charge $P_{y}$ is equated with the electric charge $q$ of the wrapped M2-brane.

\section{Quantum Dynamics}

In the previous section, we have seen that classical motions of the probes, for both M-graviton and M2-brane, all trace gyration orbits, much similar to the Landau problem. It thus brings up an issue whether, quantum mechanically, probe's excitation energy spectrum would exhibit discrete spectrum analogous to Eq.(1.2). In this section, we will find that the expectation is met precisely. We show that the energy spectrum is precisely the same as that of two copies of the two-dimensional simple harmonic oscillators, each associated with the two rotating $\mathbf{R}^{2}$-planes. 
In doing so, we will pay particular attention to the energy spectrum of the 'super-gyration orbits', which in classical analysis encompassed outside the CTC horizon. By comparing the energy spectrum with those for geodesics or 'sub-gyration orbit', one would hope to learn, if any, pathologies associated with the CTCs. Our result shows that both the energy spectrum and wave function of all probes are self-similar (Some earlier works on wave dynamics in Gödel universe include [15]).

\subsection{Graviton}

We begin with the M-theory graviton. Taking, for simplicity, the polarization entirely along $X_{6}$ direction, the field equation of a 5 -dimensional graviton $\Phi$ is given by the massless Klein-Gordon equation in the background Eq.(2.7)

$$
\square_{5} \Phi=\frac{1}{\sqrt{-g_{5}}} \partial_{m}\left(g_{5}^{m n} \sqrt{-g_{5}} \partial_{n}\right) \Phi=0 .
$$

As the Gödel metric depends only on $r_{1}$ and $r_{2}$, it is possible to decompose the field $\Phi$ into basis $\chi$ 's via separation of variables as

$$
\Phi=\left\{\chi, \chi^{*}\right\} \quad \text { where } \quad \chi=\mathcal{N} u_{1}\left(r_{1}\right) u_{2}\left(r_{2}\right) \exp \left(i L_{1} \phi_{1}+i L_{2} \phi_{2}-i E t\right) .
$$

where $\mathcal{N}$ is a normalization factor. The polar coordinates $\phi_{1,2}$ range over $[0,2 \pi]$, so the angular momenta $L_{1}, L_{2}$ are quantized to integer units. The Klein-Gordon equation Eq.(3.1) is then reduced to two coupled equations:

$$
\begin{aligned}
& {\left[-\partial_{r_{1}}^{2}-\frac{1}{r_{1}} \partial_{r_{1}}+\left(\mu E r_{1}+\frac{L_{1}}{r_{1}}\right)^{2}-E^{2} \cos ^{2} \theta\right] u_{1}\left(r_{1}\right)=0,} \\
& {\left[-\partial_{r_{2}}^{2}-\frac{1}{r_{2}} \partial_{r_{2}}+\left(\mu E r_{2}+\frac{L_{2}}{r_{2}}\right)^{2}-E^{2} \sin ^{2} \theta\right] u_{2}\left(r_{2}\right)=0 .}
\end{aligned}
$$

Here, $0 \leq \theta \leq \pi / 2$ is a parameter introduced for separation of the variables $r_{1}, r_{2}$. Eq.(13.3) takes the form of Schrödinger equation of two-dimensional simple harmonic oscillators whose natural frequency is given by $|\mu E|$ and energy by $\left\{E^{2} \cos ^{2} \theta-2 \mu E L_{1}\right\}$ or $\left\{E^{2} \sin ^{2} \theta-2 \mu E L_{2}\right\}$, respectively. Normalizable solutions are given in terms of the associated Laguerre polynomial $\mathcal{L}_{\mathbf{n}}^{\alpha}$ :

$$
\begin{aligned}
& u_{1}\left(r_{1}\right)=r_{1}^{\left|L_{1}\right|} \exp \left(-\frac{1}{2}|\mu E| r_{1}^{2}\right) \mathcal{L}_{\mathbf{n}_{1}}^{\left(\left|L_{1}\right|\right)}\left(|\mu E| r_{1}^{2}\right) \\
& u_{2}\left(r_{2}\right)=r_{2}^{\left|L_{2}\right|} \exp \left(-\frac{1}{2}|\mu E| r_{2}^{2}\right) \mathcal{L}_{\mathbf{n}_{\mathbf{2}}}^{\left(\left|L_{2}\right|\right)}\left(|\mu E| r_{2}^{2}\right)
\end{aligned}
$$

where $\mathbf{n}_{\mathbf{1}}, \mathbf{n}_{\mathbf{2}}$ are non-negative integers related to other quantum numbers as

$$
\begin{aligned}
& \mathbf{n}_{1}=-\frac{1}{2}\left(1+\left|L_{1}\right|\right)+\frac{1}{4|\mu E|}\left[E^{2} \cos ^{2} \theta-2 \mu E L_{1}\right], \\
& \mathbf{n}_{\mathbf{2}}=-\frac{1}{2}\left(1+\left|L_{2}\right|\right)+\frac{1}{4|\mu E|}\left[E^{2} \sin ^{2} \theta-2 \mu E L_{2}\right] .
\end{aligned}
$$


Adding the two radial quantum number relations in Eq.(3.5), we get

$$
E^{2}=4|\mu E|\left(\mathbf{n}_{1}+\mathbf{n}_{2}+1\right)+2\left(\mu E L_{1}+\left|\mu E L_{1}\right|\right)+2\left(\mu E L_{2}+\left|\mu E L_{2}\right|\right) .
$$

From the relation, we find the quantum energy spectrum $E$ of the M-theory graviton in a remarkably simple analytic form:

$$
|E|=4|\mu| \mathcal{N}_{0}
$$

where

$$
\mathcal{N}_{0}=\left(\mathbf{n}_{1}+\mathbf{n}_{\mathbf{2}}+1+\frac{1}{2}\left\{\left|L_{1}\right|+\operatorname{sgn}(E \mu) L_{1}\right\}+\frac{1}{2}\left\{\left|L_{2}\right|+\operatorname{sgn}(E \mu) L_{2}\right\}\right) .
$$

Thus, positive and negative branches of the energy spectrum are labelled by four non-negative integers $\mathbf{n}_{\mathbf{1}}, \mathbf{n}_{\mathbf{2}}, \mathbf{m}_{\mathbf{1}}, \mathbf{m}_{\mathbf{2}}{ }^{\dagger \dagger}$

$$
\begin{aligned}
& E^{(+)}=+4|\mu|\left(\mathbf{n}_{\mathbf{1}}+\mathbf{n}_{\mathbf{2}}+\mathbf{m}_{\mathbf{1}}^{(+)}+\mathbf{m}_{\mathbf{2}}^{(+)}+1\right), \\
& E^{(-)}=-4|\mu|\left(\mathbf{n}_{\mathbf{1}}+\mathbf{n}_{\mathbf{2}}+\mathbf{m}_{\mathbf{1}}^{(-)}+\mathbf{m}_{\mathbf{2}}^{(-)}+1\right),
\end{aligned}
$$

where $\mathbf{m}_{\mathbf{1 , 2}}{ }^{(+)}=\left(\left|L_{1,2}\right|+\operatorname{sgn}(\mu) L_{1,2}\right) / 2$ and $\mathbf{m}_{\mathbf{1 , 2}}{ }^{(-)}=\left(\left|L_{1,2}\right|-\operatorname{sgn}(\mu) L_{1,2}\right) / 2$, respectively. Notice that the two branches Eq.(3.7) of the graviton spectrum are related each other by the PT conjugation in Eq.(2.14). The energy spectrum and degeneracy is depicted in Fig. 3

A few remarks are in order.

- For massive M-graviton with mass $m$, the energy spectrum is obtainable analogously in terms of $\mathcal{N}_{0}$ in Eq.(3.6) as:

$$
E^{( \pm)}= \pm 2|\mu|\left[\mathcal{N}_{0}+\left(\mathcal{N}_{0}^{2}+\frac{1}{4} \frac{m^{2}}{\mu^{2}}\right)^{1 / 2}\right] .
$$

In Fig 3, we depict modification of the M-graviton spectrum due to non-zero mass. Notice that the energy spectrum is governed by the same orbital angular momentum quantum numbers $\mathbf{m}_{\mathbf{1}}{ }^{( \pm)}, \mathbf{m}_{\mathbf{2}}{ }^{( \pm)}$as the massless one.

- Recall that geodesics of M-graviton are closed orbits around the CTC horizon, and never travel through the CTCs. The spectrum Eq.(3.6) originates from Bohr-Sommerfeld quantization of the M-graviton wave function around the classical gyration orbit studied in section 2, so it is the counterpart of the Landau level spectrum Eq.(1.2). Orbital angular momentum of the M-graviton does not alter the interpretation, as $L_{1}, L_{2}$ are conserved quantum numbers and

\footnotetext{
${ }^{\dagger \dagger}$ The spectrum Eq.(3.7) is in agreement with the supergravity modes of the closed string spectrum in Gödel universe 4 .
} 

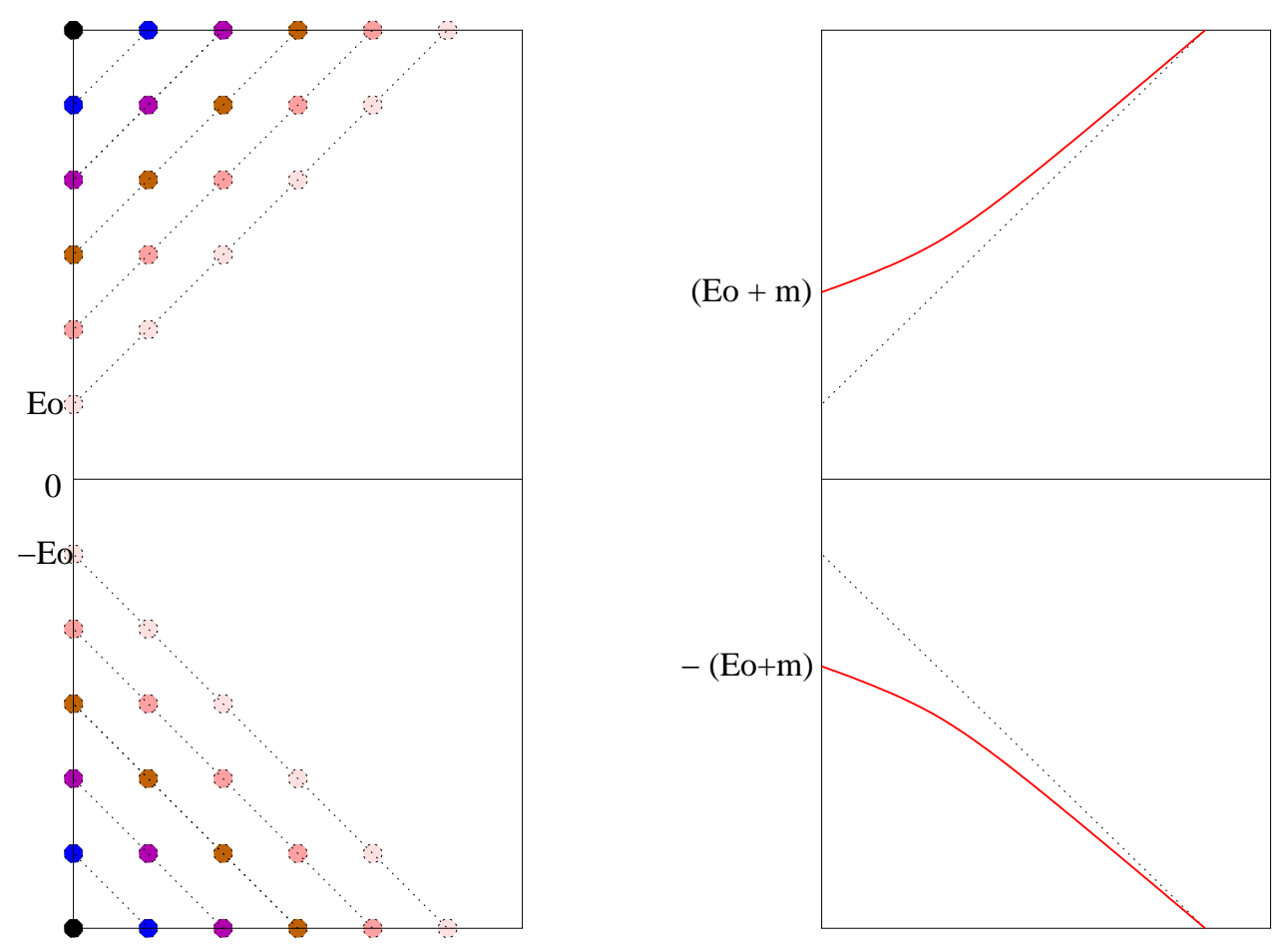

Figure 3: Energy spectrum of massless (left) and massive (right) M-theory gravitons. Horizontal axis refer to different multiplicities associated with $\mathbf{n}_{\mathbf{1}}, \mathbf{n}_{\mathbf{2}}, \mathbf{m}_{\mathbf{1}}, \mathbf{m}_{\mathbf{2}}$. The energy gap is denoted as $E_{0}=4|\mu|$.

renders the M-graviton wave equation completely separable. Notice that M-graviton's radial wave function, which determines the spatial spread-out of the M-graviton, is symmetric under reversal of the orbital angular momenta, $L_{1,2} \rightarrow-L_{1,2}$, while the energy spectrum Eq.(3.7) is not.

- The $\mathbf{n}_{\mathbf{1}}, \mathbf{n}_{\mathbf{2}}$ quantum numbers are interpretable as labelling 'excitation' above the BPS ground state of orbiting M-graviton. The zero-point energy proportional to $4|\mu|$ in Eq.(3.7) may be attributed to the fact that the null orbit is closed on each $\mathbf{R}^{2}$-plane. The frequency $|\mu|$ in Eq.(13.7) is the parameter that sets the rotation and, in turn, the CTC horizon in the Gödel universe, so it is the counterpart of the Larmor frequency $\Omega_{\mathrm{L}}$ in Eq.(1.2). Apparently, quantization of the energy spectrum has nothing to do with the presence of CNCs and CTCs in Gödel universe.

- The angular momentum quantum numbers $L_{1}, L_{2}$ run over all integer values, both positive and negative. Yet, the spectrum Eq.(3.7) is affected only when the orbital angular momentum quantum numbers take positive (negative) values, respectively, for positive (negative) energy. It implies that, for positive (negative) energy and negative (positive) orbital angular momenta, the spectrum Eq. (3.7) is infinitely degenerate. This is a direct reflection of orbit's classical behavior with nonzero orbital angular momenta. Consider a classical orbit with positive (negative) energy. As explained in section 2.3, if positive (negative) orbital angular momenta are applied to the M-graviton, orbit's diameter shrinks accordingly. It implies that, by Bohr-Sommerfeld quantization rule, quantum-mechanical energy ought to depend on the orbital angular momen- 
tum quantum numbers. For a fixed 'excitation' energy $E$ of the M-graviton, the orbital angular momentum quantum numbers cannot exceed the maximum

$$
\max \left(\mathbf{m}_{\mathbf{1}}, \mathbf{m}_{\mathbf{2}}\right)=\frac{E}{4|\mu|},
$$

and this is precisely the same upper bound $L_{\max }$ as the classical counterpart Eq. (2.24) (for $q=0$ ). Instead, if negative (positive) orbital angular momenta are applied to the M-graviton, orbit's diameter remains the same. By Bohr-Sommerfeld quantization rule, it implies that quantum-mechanical energy ought not to depend on the orbital angular momentum quantum numbers. Rather, it renders the energy level degeneracy infinite, reflecting the fact that orbit's gyration center can migrate off all over each $\mathbf{R}^{2}$-plane once the orbit angular momenta are cranked up arbitrarily high.

\subsection{M2-Brane}

Let us turn to the dimensionally reduced M2-brane. Interaction of the M2-brane with the background gauge field $G_{2}=\mathrm{d} C_{1}$ is facilitated by the minimal coupling prescription $\partial_{m} \rightarrow$ $\partial_{m}+i(q / 2 \sqrt{3}) C_{m}$ in Eq.(3.1). In the Landau gauge Eq.(2.8),

$$
C_{\phi_{1}}\left(r_{1}\right)=2 \sqrt{3} \mu r_{1}^{2} \quad \text { and } \quad C_{\phi_{2}}\left(r_{2}\right)=2 \sqrt{3} \mu r_{2}^{2}
$$

the charged and massive counterpart of Eqs.(13.3) becomes

$$
\begin{aligned}
& {\left[-\partial_{r_{1}}^{2}-\frac{1}{r_{1}} \partial_{r_{1}}+\left((E+q) \mu r_{1}+\frac{L_{1}}{r_{1}}\right)^{2}-\left(E^{2}-m^{2}\right) \cos ^{2} \theta\right] u_{1}\left(r_{1}\right)=0} \\
& {\left[-\partial_{r_{2}}^{2}-\frac{1}{r_{2}} \partial_{r_{2}}+\left((E+q) \mu r_{2}+\frac{L_{2}}{r_{2}}\right)^{2}-\left(E^{2}-m^{2}\right) \sin ^{2} \theta\right] u_{2}\left(r_{2}\right)=0}
\end{aligned}
$$

The solution is again obtainable in terms of the associated Laguerre polynomials as

$$
\begin{aligned}
& u_{1}\left(r_{1}\right)=r_{1}^{\left|L_{1}\right|} \exp \left(-\frac{1}{2}|\mu(E+q)| r_{1}^{2}\right) \mathcal{L}_{\mathbf{n}_{1}}^{\left(\left|L_{1}\right|\right)}\left(|\mu(E+q)| r_{1}^{2}\right) \\
& u_{2}\left(r_{2}\right)=r_{2}^{\left|L_{2}\right|} \exp \left(-\frac{1}{2}|\mu(E+q)| r_{2}^{2}\right) \mathcal{L}_{\mathbf{n}_{2}}^{\left(\left|L_{2}\right|\right)}\left(|\mu(E+q)| r_{2}^{2}\right)
\end{aligned}
$$

with non-negative integer quantum numbers

$$
\begin{aligned}
& \mathbf{n}_{\mathbf{1}}=-\frac{1}{2}\left(1+\left|L_{1}\right|\right)+\frac{1}{4|\mu(E+q)|}\left(\left(E^{2}-m^{2}\right) \cos ^{2} \theta-2 \mu L_{1}(E+q)\right), \\
& \mathbf{n}_{\mathbf{2}}=-\frac{1}{2}\left(1+\left|L_{2}\right|\right)+\frac{1}{4|\mu(E+q)|}\left(\left(E^{2}-m^{2}\right) \sin ^{2} \theta-2 \mu L_{2}(E+q)\right) .
\end{aligned}
$$

It then follows that the dispersion relation is given by

$$
E^{2}-m^{2}=4|\mu(E+q)| \mathcal{N}_{2}
$$


where

$$
\mathcal{N}_{2} \equiv\left(\mathbf{n}_{\mathbf{1}}+\mathbf{n}_{\mathbf{2}}+1+\frac{1}{2}\left\{\left|L_{1}\right|+\operatorname{sgn}(\mu(E+q)) L_{1}\right\}+\frac{1}{2}\left\{\left|L_{2}\right|+\operatorname{sgn}(\mu(E+q)) L_{2}\right\}\right) .
$$

The right-hand-side of Eq.(3.2) is positive-definite, and yields the two branches of the energy spectrum

$$
E^{(+)}: \quad E>m \text { and } E^{(-)}: \quad E<-m .
$$

For BPS M2-brane with $q= \pm m$, Eq.(3.2) is reduced to $E=q \pm 4|\mu| \mathcal{N}$. Keeping again track of sign of the orbital angular momentum quantum numbers, we find that the energy spectrum is given by

$$
\begin{aligned}
& E^{(+)}=q+4|\mu|\left[\mathbf{n}_{\mathbf{1}}+\mathbf{n}_{\mathbf{2}}+\mathbf{m}_{\mathbf{1}}{ }^{(+)}+\mathbf{m}_{\mathbf{2}}{ }^{(+)}+1\right] \\
& E^{(-)}=q-4|\mu|\left[\mathbf{n}_{\mathbf{1}}+\mathbf{n}_{\mathbf{2}}+\mathbf{m}_{\mathbf{1}}{ }^{(-)}+\mathbf{m}_{\mathbf{2}}{ }^{(-)}+1\right]
\end{aligned}
$$

subject to the condition Eq.(3.11). Here, $\mathbf{m}_{1,2}^{( \pm)} \equiv\left(\left|L_{1,2}\right| \pm \operatorname{sgn}(\mu) L_{1,2}\right) / 2=0,1,2, \cdots$. Remarkably, apart from the rest mass of the M2-brane, the resulting energy spectrum is identical to that of the massless M-graviton studied in the previous subsection. Notice that the energy spectra, $E^{(+)}$and $E^{(-)}$, are mapped to each other under the PT conjugation Eq.(2.14).

Several remarks are in order.

- As for the M-graviton, the energy spectrum Eq.(3.12) depends sharply on the relative sign between the energy and the orbital angular momenta. With positive (negative) orbital angular momenta of M2-brane, orbit's diameter shrinks, so the energy spectrum depends on the quantum numbers, $\mathbf{m}_{\mathbf{1}}, \mathbf{m}_{\mathbf{2}}$. For a fixed 'excitation' energy $E$ of the M2-brane, these quantum numbers cannot exceed the maximum

$$
\max \left(\mathbf{m}_{1}, \mathbf{m}_{\mathbf{2}}\right)=\frac{(E-q)}{4|\mu|}
$$

and this is precisely the upper bound $L_{\max }$ of the classical counterpart Eq.(2.24) (for $q \neq 0$ ). With negative (positive) orbital angular momenta of the M2-brane, classical orbit's diameter remained unaffected, and the energy spectrum is independent of $\mathbf{m}_{\mathbf{1}}, \mathbf{m}_{\mathbf{2}}$ quantum numbers. Instead, the energy levels become infinitely degenerate, reflecting classical result that orbit's gyration center can migrate off all over each $\mathbf{R}^{2}$-plane once M2-brane's orbit angular momenta are cranked up arbitrarily high.

- Classically, we learned in section 2 that, unlike M-graviton, M2-brane probe can pass through the CTC horizon and gyrate around in the region where the co-moving observer at $O$ perceives CTCs, even for zero orbital angular momenta. Quantum mechanically, wave function and hence probability density of the two probes Eqs.(3.4) and Eqs.(3.8), respectively, are self-similar. The 
two are merely related each other by (energy-dependent) rescaling radial coordinates by an energy-dependent factor $\sqrt{1+(q / E)}$. Moreover, for both, the energy scale of the excitation spectrum is set by one and the same Larmor frequency, $4|\mu|$. It may be that, in order for the quantum mechanics to reveal full-fledged pathologies of the CTCs present, more refined dynamical processes, e.g. interference of multi-body wave functions or nonlinear mode-mode interactions, should be considered.

- Recall that, in the foregoing analysis, we have tacitly assumed that the orientation of the two-cycles M2-brane wraps on is such that the M2- and $\overline{\mathrm{M} 2}$-branes carry electric charge $q$ positive and negative, respectively. Upon reversing orientation of the two-cycles $\ddagger$, the sign of the electric charge $q$ would flip. All the foregoing analysis would be the same except that $q$ should be taken the opposite. Still, as $E>m$ and $E<-m$, the spectrum is isomorphic to the above.

\subsection{IIB String Theory Setup}

By taking similar steps, one can find excitation energy spectrum of IIB-graviton probe by solving the six-dimensional wave equation, $\square_{6} \Phi=0$, in the background Eq.(2.26). Making use of the conserved quantum numbers identified in section 2.4, we again take separation of variables as

$$
\Phi=\left(\chi, \chi^{*}\right) \quad \chi=\mathcal{N} u_{1}\left(r_{1}\right) u_{2}\left(r_{2}\right) \exp \left(i L_{1} \phi_{1}+i L_{2} \phi_{2}+i P_{y} y-i E t\right)
$$

Radial parts of the wave equation are then given by

$$
\begin{aligned}
& {\left[-\partial_{r_{1}}^{2}-\frac{1}{r_{1}} \partial_{r_{1}}+\left(\left(E-P_{y}\right) \mu r_{1}+\frac{L_{1}}{r_{1}}\right)^{2}-\left(E^{2}-P_{y}^{2}\right) \cos ^{2} \theta\right] u_{1}\left(r_{1}\right)=0} \\
& {\left[-\partial_{r_{2}}^{2}-\frac{1}{r_{2}} \partial_{r_{2}}-\left(\left(E-P_{y}\right) \mu r_{2}+\frac{L_{2}}{r_{2}}\right)^{2}-\left(E^{2}-P_{y}^{2}\right) \sin ^{2} \theta\right] u_{2}\left(r_{2}\right)=0 .}
\end{aligned}
$$

One finds that they are the same as the ones for the M-graviton and the M2-brane for $P_{y}=0$ and $P_{y}=-q$, respectively, so the wave function and energy spectrum are exactly the same as those.

\section{Discussion}

In this paper, we have studied classical and quantum dynamics of M-graviton and M2-brane probes in Gödel universe, with particular attention to possible effects of the CTCs present beyond the CTC horizon of a co-moving observer. Our results are summarized as follows.

\footnotetext{
$\ddagger \ddagger$ Orientation of the normal directions needs to be reversed concurrently so that the net orientation of $X_{6}$ is unaffected.
} 
- On each rotating $\mathbf{R}^{2}$-plane, all probes trace gyration orbits, much similar to the Landau problem, Eq.(1.1). For each orbit, gyration center and diameter depends on probe's conserved quantum numbers: energy $E$, angular momenta $L$, and 3 -form potential charge $q$.

- For zero orbital angular momenta, orbit of M-graviton (geodesics) traces radial distance between a co-moving observer located at origin $O$ and the CTC horizon $R_{\mathrm{CTC}}$ perceived by the co-moving observer. Orbit of M2-brane traces between a co-moving observer at $O$ and the maximal distance $D$ (diameter of the orbit). For $\operatorname{sgn}(E q)>0, D$ is less than $R_{\mathrm{CTC}}$, so as perceived by the co-moving observer, the orbit does not travel through the region where CTCs are present. For $\operatorname{sgn}(E q)<0, D$ is larger than $R_{\mathrm{CTC}}$, and the orbit travels through the region where CTCs are present.

- Nonzero orbital angular momenta $L$ modify probe's orbit as well. For $\operatorname{sgn} \mu L>0$, the gyration takes place at the same center as $L=0$ but the gyration diameter is shrunken. For $\operatorname{sgn} \mu L<0$, the gyration diameter is the same as $L=0$ but the center migrates away from the co-moving observer.

- Quantum mechanically, probe's wave functions and excitation energy spectrum are all selfsimilar, independent of whether probe's classical orbit is larger or smaller than the CTC horizon. Interestingly, under the reversal of the orbital angular momentum, the wave functions are invariant.

Our results indicate that further investigation is imperative for exploring full-fledged pathology of the Gödel universe in M-theory. It is evident that naive application of quantum field theory approximation to M-theory on Gödel universe is ill-defined. For example, because Gödel universe is not globally hyperbolic, inner products is ill-defined. As such, expectation value of the energy-momentum tensor $\left\langle T_{m n}\right\rangle$ and back-reaction thereof are not computable.

An attitude one may take for the Gödel universe is that it is similar to negative-mass Schwarzschild or extremal Reissner-Nordstrom black hole. Formally, the latter is a solution with a naked time-like singularity, repelling all time-like geodesics. For both negative-mass black holes and Gödel universe, they are not likely to be formed out of physical processes in initial space-time without pathologies such as CTCs or singularities. Supersymmetries preserved by the Gödel universe would not help much as the negative-mass extremal Reissner-Nordstrom black hole as well is embeddable as a supersymmetry preserving configuration. Our tentative attitude is though sympathetic to [9]: until proven inconsistent, the Gödel universe in M-theory deserves further inspection, including possible observational constraints [16].

\section{Acknowledgement}

We acknowledge Eric Gimon, Rajesh Gopakumar, Carlos Herdeiro, Gary Horowitz, Oleg Lunin, Juan Maldacena, Fumihiko Sugino, Hiromitsu Takayanagi and Tadashi Takayanagi for enlight- 
ening discussions. This work was done while SJR was a Member at the Institute for Advanced Study. He thanks the School of Natural Sciences for hospitality and for the grant in aid from the Fund for Natural Sciences.

\section{Appendix}

\section{Five-Dimensional Gödel metric}

In the bipolar coordinates, nonzero components of the inverse metric are

$$
\begin{aligned}
& g^{t t}=-1+\mu^{2}\left(r_{1}^{2}+r_{2}^{2}\right) \\
& g^{r_{1} r_{1}}=g^{r_{2} r_{2}}=1, \\
& g^{\phi_{1} \phi_{1}}=\frac{1}{r_{1}^{2}} \quad g^{\phi_{2} \phi_{2}}=\frac{1}{r_{2}^{2}} \quad g^{\phi_{1} t}=g^{\phi_{2} t}=-\mu .
\end{aligned}
$$

Nonzero components of the Christoffel connection are:

$$
\begin{array}{lll}
\Gamma_{r_{1} t}^{t}=\mu^{2} r_{1} & \Gamma_{\phi_{1} t}^{r_{1}}=\mu r_{1} & \Gamma_{r_{1} t}^{\phi_{1}}=-\mu / r_{1} \\
\Gamma_{\phi_{1} r_{1}}^{t}=\left(\mu r_{1}\right)^{3} & \Gamma_{\phi_{1} \phi_{1}}^{r_{1}}=-r_{1}\left(1-2 \mu^{2} r_{1}^{2}\right) & \Gamma_{\phi_{1} r_{1}}^{\phi_{1}}=-\mu^{2} r_{1}+1 / r_{1} \\
\Gamma_{\phi_{2} r_{1}}^{t}=\mu^{3} r_{1} r_{2}^{2} & \Gamma_{\phi_{1} \phi_{2}}^{r_{1}}=\mu^{2} r_{1} r_{2}^{2} & \Gamma_{\phi_{2} r_{1}}^{\phi_{1}}=-\mu^{2} r_{2}^{2} / r_{1} \\
\Gamma_{r_{2} t}^{t}=\mu^{2} r_{2} & \Gamma_{\phi_{2} t}^{r_{2}}=\mu r_{2} & \Gamma_{r_{2} t}^{\phi_{2}}=-\mu / r_{2} \\
\Gamma_{\phi_{2} r_{2}}^{t}=\left(\mu r_{2}\right)^{3} & \Gamma_{\phi_{2} \phi_{2}}^{r_{2}}=-r_{2}\left(1-2 \mu^{2} r_{2}^{2}\right) & \Gamma_{\phi_{2} r_{2}}^{\phi_{2}}=-\mu^{2} r_{2}+1 / r_{2} \\
\Gamma_{\phi_{1} r_{2}}^{t}=\mu^{3} r_{1}^{2} r_{2} & \Gamma_{\phi_{1} \phi_{2}}^{r_{2}}=\mu^{2} r_{1}^{2} r_{2} & \Gamma_{\phi_{1} r_{2}}^{\phi_{2}}=-\mu^{2} r_{1}^{2} / r_{2}
\end{array}
$$

\section{Six-Dimensional Gödel Universe}

For the six-dimensional Gödel universe $\mathcal{G}_{6}$ in Type IIB string theory, non-zero components of the inverse metric are

$$
\begin{aligned}
& g^{t t}=-1+\mu^{2}\left(r_{1}^{2}+r_{2}^{2}\right) \quad g^{\phi_{1} t}=g^{\phi_{2} t}=g^{y \phi_{1}}=g^{y \phi_{2}}=-\mu \quad g^{y t}=\mu^{2}\left(r_{1}^{2}+r_{2}^{2}\right), \\
& g^{r_{1} r_{1}}=g^{r_{2} r_{2}}=1 \quad g^{\phi_{1} \phi_{1}}=\frac{1}{r_{1}^{2}} \quad g^{\phi_{2} \phi_{2}}=\frac{1}{r_{2}^{2}} \quad g^{y y}=1+\mu^{2}\left(r_{1}^{2}+r_{2}^{2}\right),
\end{aligned}
$$

and non-zero components of the Christoffel connection are

$$
\begin{aligned}
& \Gamma_{r_{1} t}^{t}=\Gamma_{r_{1} t}^{y}=-\Gamma_{r_{1} y}^{y}=-\Gamma_{r_{1} y}^{t}=\mu^{2} r_{1} \quad \Gamma_{\phi_{1} t}^{r_{1}}=-\Gamma_{\phi_{1} y}^{r_{1}}=\mu r_{1} \\
& \Gamma_{\phi_{1} \phi_{1}}^{r_{1}}=-r_{1} \quad \Gamma_{\phi_{1} r_{1}}^{\phi_{1}}=\frac{1}{r_{1}} \quad \Gamma_{r_{1} t}^{\phi_{1}}=-\Gamma_{r_{1} y}^{\phi_{1}}=-\frac{\mu}{r_{1}}
\end{aligned}
$$

along with those replaced with $(1 \leftrightarrow 2)$.

\section{References}

[1] K. Gödel, An example of a new type of cosmological solutions of Einstein's field equations of gravitation, Rev. Mod. Phys. 21, 447 (1949). 
[2] S.W. Hawking, The chronology protection conjecture, Phys. Rev. D 46, 603 (1992).

[3] J.P. Gauntlett et.al., All supersymmetric solutions of minimal supergravity in fivedimensions, arXiv:hep-th/0209114.

[4] T. Harmark and T. Takayanagi, Supersymmetric Goedel universes in string theory, arXiv:hep-th/0301206.

[5] G.W. Gibbons and C. Herdeiro, Supersymmetric rotating black holes and causality violations, Class. Quant. Grav. 16, 3619 (1999) arXiv:hep-th/9906098.

[6] C.A. Herdeiro, Special properties of five-dimensional BPS rotating black holes, Nucl. Phys. B582, 363 (2000) arXiv:hep-th/0003063; ibid. Spinning deformations of the D1-D5 system and a geometric resolution of closed timelike curves arXiv:hep-th/0212002.

[7] L. Dyson, Chronology Protection in string theory arXiv:hep-th/0302052.

[8] R. Biswas, E. Keski-Vakkuri, R.G. Leigh, S. Nowling and E. Sharpe, The taming of closed timelike curves arXiv:hep-th/0304241.

[9] E. K. Boyda, S. Ganguli, P. Horava and U. Varadarajan, Holographic protection of chronology in universes of the Goedel type, arXiv:hep-th/0212087.

[10] D. Bak and S.-J. Rey, Cosmic Holography, Class. Quant. Grav. 17, L83 (2000) hep-th/9902173.

[11] R. Bousso, Holography in General Space-Times, JHEP 9906 (1999) 028 arXiv:hep-th/9906022.

[12] N. Drukker, B. Fiol and J. Simón, Gödel's Universe in a Supertube Shroud arXiv:hep-th/0306057.

[13] S. Chandrasekhar and J.P. Wright, The geodesics in Gödel universe, Proc. Nat. Acad. Sci. 47, 341 (1961).

[14] Work in progress.

[15] B. Mashhoon, Influence of gravitation on the propagation of electromagnetic radiation, Phys. Rev. D 11, 2679 (1975);

W. A. Hiscock, Scalar perturbations in the Godel universe, Phys. Rev. D 17, 1497 (1978).

[16] E.F. Bunn, P. Ferreira and J. Silk, How anisotropic is our universe?, Phys. Rev. Lett. 77, 2883 (1996) astro-ph/9605123. 